\title{
A simple synthesis of magnetic nanoparticles-supported 4- aminomethylbenzoic acid as a highly efficient and reusable catalyst for synthesis of 2-amino-4Hchromene derivatives
}

\author{
Mohammad Mehdi Khodaei ${ }^{1,2}$, Abdolhamid Alizadeh $^{1,2}$, Maryam Haghipour ${ }^{1}$
}

${ }^{1}$ Department of Chemistry, Razi University, Kermanshah 67149-67346, Iran

${ }^{2}$ Nanoscience and Nanotechnology Research Center (NNRC), Razi University, Kermanshah 67149-67346, Iran

\begin{abstract}
A new 4-aminomethylbenzoic acid-functionalized Fe3O4 magnetic nanoparticles as a hybrid heterogeneous catalyst was synthesised and characterized by FT-IR, XRD, TGA, TEM, SEM and VSM techniques. The catalytic activity of this nanocatalyst was probed through one-pot synthesis of 2-amino-4H-chromene derivatives from three component reactions of various aldehydes, malononitrile and dimedone. The chemically and thermally stable catalyst was easily recovered using an external magnet and reused for at least five successive runs without significant loss of its activity. The simplicity of the method, high yields of the products, mild reaction conditions and low reaction times are the other advantages of this procedure.
\end{abstract}

Keywords: Nanoparticle, Heterogeneous catalyst, 4-Aminomethylbenzoic acid, 2-Amino-4Hchromene, Aldehydes, Dimedone. 